\title{
A Semi-Physical Simulation Experiment System for Automatic Control Education
}

\author{
Liu Zhong, Yuan Shaoqiang \\ School of Automation Science and Electrical Engineering \\ Beihang University \\ Beijing, P.R.China \\ lzpro@126.com, ysq1960@sina.com
}

\begin{abstract}
Automatic control theory is an important professional course of the automation and correlation specialties. This course is not pure theoretical, but practical for its close relationship with practical industrial automation systems design and implementation. Experiment plays an irreplaceable role in the process of the whole course learning. Most of traditional experiments for automatic control theory are pure simulated process. Furthermore, the experiment items are mainly demonstrated or confirmatory. This sort of experiment can be considered as another kind of class exercise. Students cannot get knowledge and technical ability in this way. A hardware-in-the-loop simulation experiment system based on MATLAB/RTW (Real-Time Workspace) is introduced in this paper. The software is open sources based on MATLAB. The hardware includes a PCI multi-purpose data acquisition card and an experimental box including analog circuit. According to the experiences of more than 5 years application, this semi-physical system is effective to attract student's interests. And through laboratory experiments education, understanding of the theory concept and practical ability are improved. An experiment teaching method based on experiential learning theory is also introduced.
\end{abstract}

Keywords-automatic control; matlab; real-time workshop; experiential learning; experiment

\section{INTRODUCTION}

For the need of cultivating student's ability of innovation and engineering, experiment teaching has received more and more concentration in recent years. Experiment is a bridge over concept connecting theory and practice. Almost all abstract theories can be demonstrated through simple experiments. Besides, a learner can integrate his knowledge and skills in the process of experiment to comprehend theory and find new problems. In some sense, experiment teaching has more advantages than lecture teaching for its tightly relationship with practice. For the latest few decades, automatic control has flourished as a major and successful field in almost every engineering subject [1]. Automatic control theory is a core foundation professional course of automation specialty and part of the respective engineering curricula. But the course is hard to be mastered for its abstract and integration of related courses, such as advanced mathematics, linear algebra, complex variable functions, etc. Furthermore, automatic control is closely related to practical industrial automation systems. It is not only theoretical, but also practical. The whole teaching process should integrate classroom lecture with laboratory experiment practice. Application of experiments in the process of teaching is considered as an effective approach. Most of laboratory experiments for automatic control theory are pure simulated processes. The experiment items are mainly demonstrated or confirmatory. Actually, this traditional learning style is not suitable for undergraduate students. They have relatively good capability for solving problems through several years' study and practice. Students incline to consider the experimental results as the only purpose and obtain data through all kinds of methods including non-academic means. Theory and concept of the course are neglected and the experimental process does not really deepen understanding of theoretical principles.

In this paper, a hardware-in-the-loop simulation experiment system based on MATLAB/RTW is proposed. Teaching reform of laboratory experiment education for automatic control theory is also introduced.

\section{CURRENT SitUATION OF LABORATORY}

Automatic control theory is the most important professional course for the students of automation specialty. Over 10 specialties students take this course in Beihang University. Teaching and Experiment Center for Automation and Testing is responsible for all laboratory experiment teaching of automatic control theory. Our center takes up more than 80 classes and the total number of students is not less than 2400 ever year. Our laboratory hall can accommodate 35 students working independently with his/her own experiment system.

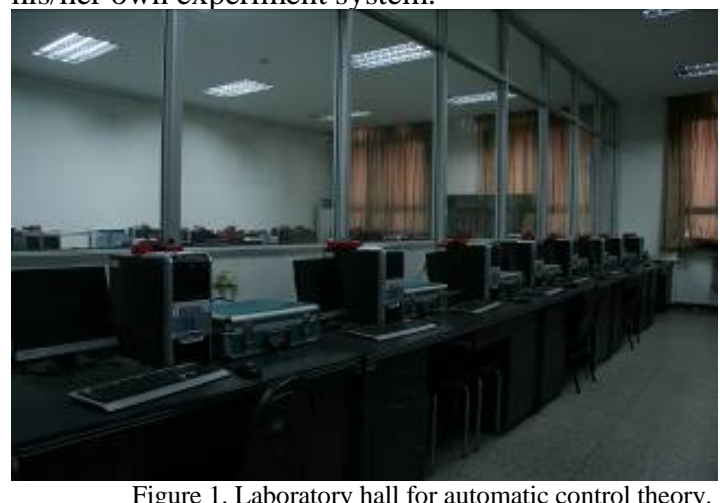

The whole experiment for automatic control consists of seven relatively independent components.

- $\quad$ step response analysis of first-order and secondorder system

- frequency domain analysis of linear system

- $\quad$ series correction 
- digital simulation of control system

- research on sampling system

- $\quad$ state feedback and state observer

- response of dynamic process for nonlinear component

Each component includes two class hours' experiment. These experiments are tightly related to theory sessions and most of them are demonstrated or confirmatory. Every experiment is well designed in such a way that the theoretical principles can be demonstrated.

\section{SYSTEM ARCHITECTURE}

\section{A. System Structure}

Laboratory experiment system can be divided into three categories including pure simulation system, pure physical system and semi-physical simulation system. In pure simulation system, students do not need concern the details of the system. If students change any active element in simulation software an immediate recalculation and presentation automatically begins [2][6]. In this way, they perceive how their modifications affect the result obtained. The advantage of this system is simple and low cost. The same parameters will get the same result and correct parameters will get right result whatever students do in the experiment. This process is similar to homework exercise for its content being parameters calculation. Pure physical experiment is more similar to practical engineering. All equipments are physical devices which can develop the student's engineering intuition. But this system is always costly and with difficulties in developing and maintaining. Furthermore, students have to concentrate on device details and need more time to familiar with practical system.

From the above analysis, we adopt a semi-physical simulation system including a personal computer and an analog circuit experimental box [3]. This type of system is easy to be maintained with low cost. The control object is an analog circuit constructed by student himself. The simulation part of the system is a digital controller implemented by computer using MATLAB/RTW. A multi-purpose data acquisition card is adopted for real time data acquisition and analog signal output. Figure 2 shows the structure of the proposed system.

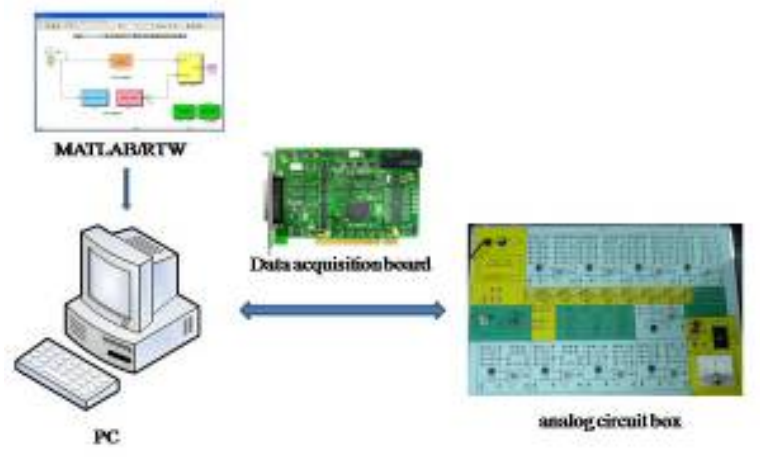

Figure 2. Structure of the proposed system.

\section{B. Software and Hareware Design}

Real-time performance is a key feature of the semiphysical system for the application of practical devices. Sampling and controller output must keep up with the changes of physical components. MATLAB is a programming environment for a wide range of applications, such as algorithm development, data analysis, simulation, and so on [7]. SIMULINK provides a powerful and versatile simulation platform integrated with MATLAB [8]. In order to enhance the generality of the SIMULINK program, transformation from SIMULINK models into other programming languages is supported through the integrated component RTW. SIMULINK models can be easily ported into other simulation platforms, especially for the real-time simulation platforms. The controller and correlative components are constructed by SIMULINK models and hardware interface between MATLAB and data acquisition device is set. This SIMULINK model is open source. Students can easily modify parameters of controller, sources and other components and change the system structure without writing any program. The result can be acquired through scope component in SIMULINK. Furthermore, the experiment model is designed two separately routes. The upper one is pure digital simulation; the lower one is semiphysical simulation as illustrated in figure 3 . The results can be compared by the response curve.

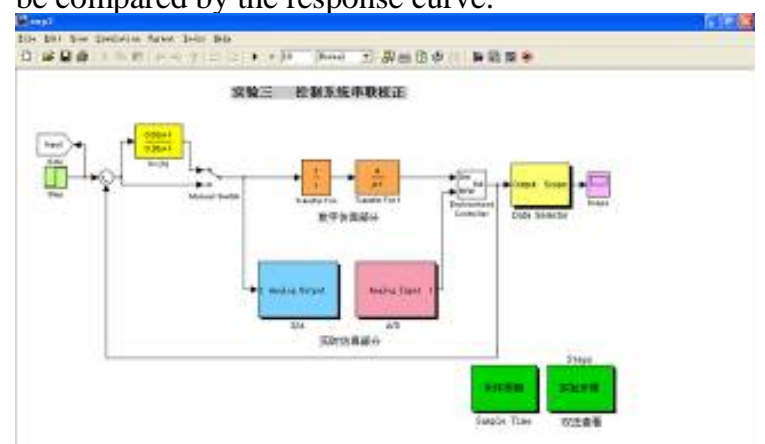

Figure 3.Interfac of experiment 3: series correction.

On account of cost reducing, a general data acquisition card was adopted and device driver for MATLAB was developed. This card concludes eight 16-bit A/D converters and two D/A converters. The response of SIMULINK model is acquired by $\mathrm{A} / \mathrm{D}$ converter and input is supplied by $\mathrm{D} / \mathrm{A}$ converter. This approach established seamless connection between SIMULINK and practical devices. Students need not know the details of interface between hardware and software.

Hardware component is an analog circuit box with nine independent operational amplifier units. Figure 4 shows one of the nine op-amp units on experimental box. Experiment circuit is constructed by students himself using test wires. In most experiments, the control object is established through this method. 


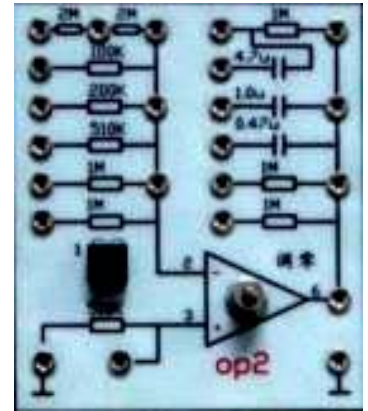

Figure 4.An op-amp unit on experimental box.

Over several years' application of the experiment system, more than 7000 students finished their automatic control theory courses. This semi-physical experiment system is more concrete and flexible than former pure simulation system for its physical components. The system provided more space for students to do anything they want and realize easily through MATLAB environment or alterable analog circuits. Figure 5 shows the whole experiment system.

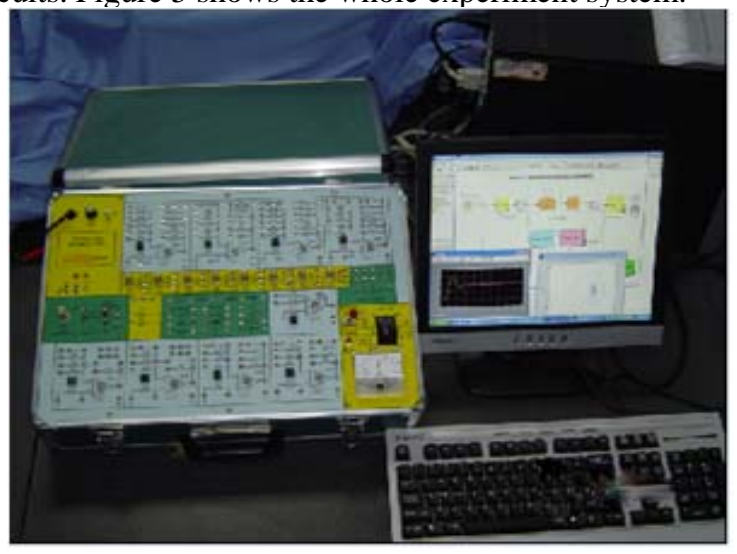

Figure 5. Semi-physical experiment system.

\section{REFORM OF EXPREIMENT TEACHING}

Although the proposed semi-physical experiment system has done well effect on comprehension of concept and theory understanding, there are still some defects in the process of experiments. Most students always take experiments without preparation, and all what they do is according to detailed experimental guiding books. The following three problems should be noticed.

- Most of experiments are demonstrated or confirmatory. The process lacks free space for innovation.

- There are too many details in construction book. Most students can easily finish the experiment according to step by step instructions.

- The process of experiment teaching is similar to lecture teaching.

Experiment was always considered as a minor part of lecture course. The purpose of execute experiment is only to demonstrate the theory or concept in lecture course. The underlying mean of experiment is not regarded. According to psychology research, learning process starts from experience and knowledge is amended through practical application.
Experiment is not only a demonstration process, but also theory learning process. Knowledge is created through the transformation of experience. Knowledge is result from the combination of grasping and transforming experience [4]. According to Kolb's experiential learning theory, learning process includes four basic stages: Concrete Experience (CE), Reflection Observation (RO), Abstract Conceptualization (AC) and Active Experimentation (AE).

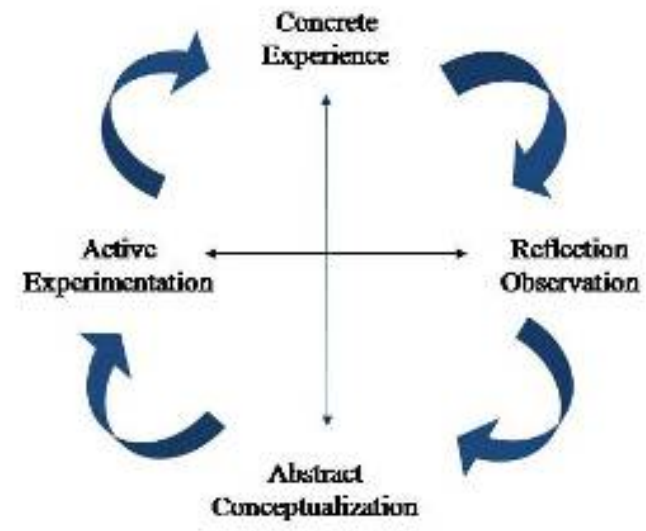

Figure 6. Kolb’s learning cycle.

During the laboratory experiment session, students are mainly involved in the active experimentation stage according to Kolb's learning cycle. But laboratory experiment is not the final session. Students should amend his knowledge and start a new learning cycle. Experiment should not be considered as an isolated process in learning cycle.

To solve above problems in experiment, some reforms were adopted to improve the quality of experiment teaching. First, some experiment sessions are changed to be comprehensive and designing. And some industrial practical control methods are adopted. For example, series correction is a confirmatory experiment formerly. A design session is adopted to calculate the parameters of controller according definite index. Students have to understand the meaning of parameters to finish the experiment. Proportional-integralderivative (PID) controller design is also included in this session for its widely application in automation of industrial processes. PID control is one of the most useful control algorithms in linear and nonlinear control systems [5]. In theory classes, this important controller is always lectured briefly for the lack of class hour. In theory, this part is not important and abstract. But it is very important in practical application. Each component has definite meanings and specific effect on control result. Students will deepen comprehension of correction through adjusting parameters.

Traditional instruction book is written step by step in details. It can help students to find what they should do clearly. But this mode will restrict student's mind in a fix pattern. A new style of instruction book is designed for freedom of thinking. The instruction book does not tell students what they should do in detail. Task and target is the only important thing. The book only provides necessary information and technology support. All experiment process is designed by students themselves. 
Experiment teaching process follows up the style of lecture teaching. At the beginning of experiment class, lecturer always introduces some theoretical information and experimental procedures. Actually, experiment is not extension of lecture teaching for its unique characteristics. Knowledge and ability should be acquired through self experiences in experiment class. Experience is the first thing student should do in experiment class. If some problems occur in the process of experience, they can be solved by discussion and any other methods. This teaching mode makes student as leading role in learning process and stresses the effect of experience.

\section{CONCLUSION}

The designed and application of semi-physical simulation experiment system is proposed in this paper. MATLAB/RTW is adopted for its real-time performance. The software structure is designed using SIMULINK for its flexible application. The proposed experiment system can directly generate real-time control program, parameters can be modified online and control effect can be observed immediately using SIMULINK graphic model. Students can focus on theoretical designing and concept understanding without concerning irrespective details. For the problems of automatic control theory experiment, teaching reform is applied based on Kolb's experiential learning theory. The effect of experience is emphasized.

\section{ACKNOWLEDGMENT}

This work was supported by Construction of Teaching Teams Project and Education Reform Project, Beihang University

\section{REFERENCES}

[1] SD.Bencomo, "Control learning: present and future”,Annual Reviews in control ,2004,pp.115-136.

[2] V. Kroumov and H. Inoue, "Enhancing education in automatic control via interactive learning tools", Proc. SICE Annu Conf(SICE2001), Jul.2001, pp.220-225.

[3] Yuan Shaoqiang, Chen LiLi, Xingshan, "Study and application of semi-physical simulation condition based on RTW”, Chinese Journal of Scientific Instrument, 2007,27(6): 2585-2586

[4] Kolb, D, A, "Experiential Learning: Experience as the Source of Learning and Development”, New Jersey: Prentice-Hall, 1984.

[5] R, Kelly, J.Moreno,"Learning PID structures in an introductory course of automatic control”, IEEE Transactions on education, Nov.2001,pp373-376.

[6] M.Casini, D.Prattichizzo,A.Vicino,’The automatic control telelab: a user-friendly interface for distance learning”, IEEE.tranctions on education, May,2003,pp252-257.

[7] Kai Zheng, Yu Yao, Yi Jiang, Ruqi Wang, “A new real-time multitasking simulation scheme based on MATLAB RTW and RTX”,Coference on Intelligent Control and Information Processing, July 2012, pp185-190.

[8] MATLAB: Users Guide., Nattick, MA: The MathWorks Staff and MathWorks, Inc., 2009. 